Revue d'histoire de l'Amérique française

REVUE D.HISTOIRE DE L'AMÉRIQUE FRANÇAISE

\title{
Les plans en relief de Québec, Montréal et Louisbourg
}

\section{René Baudry}

Volume 16, numéro 2, septembre 1962

URI : https://id.erudit.org/iderudit/302194ar

DOI : https://doi.org/10.7202/302194ar

Aller au sommaire du numéro

Éditeur(s)

Institut d'histoire de l'Amérique française

ISSN

0035-2357 (imprimé)

1492-1383 (numérique)

Découvrir la revue

Citer cet article

Baudry, R. (1962). Les plans en relief de Québec, Montréal et Louisbourg. Revue d'histoire de l'Amérique française, 16(2), 213-218.

https://doi.org/10.7202/302194ar d'utilisation que vous pouvez consulter en ligne.

https://apropos.erudit.org/fr/usagers/politique-dutilisation/ 


\section{LES PLANS EN RELIEF DE QUÉBEC, MONTREAAL ET LOUISBOURG}

Louis XIV avait décidé en 1668, à la suggestion de Louvois, son ministre de la guerre, la création d'une collection de modèles en relief des places fortes du royaume. Ces représentations devaient servir à étudier «de visu » les ouvrages nécessaires à la défense des villes frontières, et même à préparer certains projets de conquête, puisqu'il avait aussi fait lever les plans de quelques villes des pays voisins ! Vauban, qui dirigeait les fortifications, employa plusieurs ingénieurs à fabriquer une cinquantaine de ces plans.

Le Roi y attachait une grande importance, puisqu'il les fit installer au premier étage du Louvre, dans la Grande galerie et en interdisait l'accès au public, comme secret de guerre. Une miniature du temps, par Van Blarenberghe, montre le souverain et ses ministres en train d'examiner les plans dans cette immense salle.

Aucun plan des colonies n'y figurait encore, mais Vauban devait y songer, puisqu'un de ses protégés écrivait dans un mémoire à Pontchartrain: "Le sieur de Villeneuve avait dessin (sic) avant son départ de Canada de faire des plans en relief de Québec, les Trois Rivières et le Montréal, à la manière du Sieur de Montaigu, avec lequel il a travaillé en France, pour instruire parfaitement Sa Majesté de l'état des lieux. Mais ses appointements suffisant à peine pour vivre ne lui ont pas permis d'en faire les avances. Si votre Grandeur souhaite, il y travaillera. ${ }^{1}$

Après la mort de Louis XIV, le Conseil de Marine continua de s'intéresser à cette collection, et l'étendit aux colonies. L'ingénieur Chaussegros de Léry reçut l'ordre d'exécuter les reliefs de Québec et de Montréal. Il les fit dresser en 1720 et 1721, et

1 Mémoire de Villeneuve sur les fortifications de Québec, adressé à Pontchartrain, en 1691. Dépôt des Fortifications des Colonies, Amérl. sept., No 355 , p. 6. 
la correspondance officielle garde mention du coûteux transport de ces pièces encombrantes jusqu'au Havre, puis de là par la Seine jusqu'à Rouen et Paris. ${ }^{2}$ Le relief de Louisbourg dut être exécuté un peu plus tard.

Sous Louis XV et Louis XVI, la collection continua de s'accroître. Elle dépendait du ministère de la guerre, mais l'entretien des locaux relevait de l'administration des bâtiments. Le vaste espace qu'occupaient ces maquettes gênait les administrateurs, qui négligèrent d'abord l'entretien des locaux, puis obtinrent leur départ. Elles furent transportées aux Invalides. Le déménagement dura six mois, et certains plans, construits sur place, étaient si considérables qu'il fallut ouvrir le toit, afin de pouvoir les sortir. La négligence et le déménagement avaient beaucoup endommagé les reliefs, et il fallut travailler plusieurs années à leur restauration. Un croquis de 1777 nous donne un aperçu de la nouvelle installation aux Invalides. La collection comprenait alors 115 plans, et les trois plans canadiens, groupés ensemble, occupaient l'angle nord-est de la salle, près de l'entrée. Pour visiter, il fallait toujours la permission écrite du Roi, et la dernière autorisation fut accordée à Rochambeau et sa suite par Louis XVI, le 23 mars 1792, moins d'un an avant sa mort.

Sous la Révolution, la collection fut rattachée au Dépôt des Fortifications de la Guerre. Napoléon s'y intéressa activement et fit dresser de nouveaux plans, d'après des levées topographiques précises et des méthodes rigoureusement scientifiques. Mais peu à peu la stratégie évolua, et ces plans perdirent leur utilité militaire, pour ne conserver qu'une valeur historique. A partir de 1799, le public put les admirer, dans des expositions annuelles. La collection, rattachée au Service géographique de l'armée en 1885 , fut exposée régulièrement depuis 1920 , et classée en 1927 comme monument historique. Elle dépend maintenant du ministère de l'Éducation nationale et occupe une partie des combles, au quatrième étage, dans la partie est de l'Hôtel des Invalides. Une moitié seulement des plans est visible; les autres attendent une restauration.

2 Voir J.-Edmond Roy, Rapport sur les Archives de France ... (Ottawa, 1911), 542-44. 
Mais les plans canadiens de Québec, Montréal et Louisbourg n'y figurent plus. Que sont-ils devenus ? M. Arthur G. Doughty entreprit, vers 1903 , une enquête à ce sujet, ${ }^{3}$ reprise plus tard par M. J.-Edmond Roy. Ils ne purent localiser ces plans. Dès 1854 cependant, M. P.-L. Morin, envoyé en France par le Ministère des Terres de la Couronne, avait noté dans son rapport: «Dans les combles de l'Hotel des Invalides, découverte des plans en relief des villes de Québec, Montréal et Louisbourg ».4 Ces plans existaient donc encore en 1853. Cette indication permettait d'espérer les retrouver, sinon dans les salles, au moins dans les réserves du Musée des plans en relief. M. Grodecki, conservateur actuel de ce Musée, a eu l'amabilité de procéder à quelques recherches et, grâce à lui, nous avons pu connaître la destinée de ces plans, depuis leur entrée aux Invalides. En effet, les inventaires périodiques du Musée les mentionnent avec leurs numéros d'ordre, en 1777,1790 et 1794 . En 1810, ils devaient présenter une assez piteuse mine, puisqu'on les retira des salles d'exposition et qu'on les mit en entrepôt. Le "Tableau des plans en magasin 》 de 1811 décrit ainsi les deux premiers:

Plan de la ville de Québec..., représenté à marée basse, avec les fortifications proposées et approuvées, fait par le Sr Chaussegros de Léry, le 30 sept. 1720. Rectangulaire, 8 p. $/ 100$ t. Dimensions: $2.80 \times 2.20$. Fond en bois, relief en carton. En mauvais état, à réparer.

Montréal..., avec les fortifications projetées et approuvées. Fait au Montréal par Chaussegros de Léry, le 15 mai 1721. Signé. Parallélogramme, 2.40 $x$ 1.90. $8 \mathrm{p} / 100 t$. Fond de bois, terrain en carton, maisons en bois. En mauvais état; beaucoup de maisons manquent. A réparer.

Le «Tableau» de 1871 décrit ainsi le plan de Louisbourg:

Relief de la partie méridionale du port de Louisbourg, au sud de l'Ile Royale, avec les projets de fortifications. Rectangle, $1.90 \times 1.60$. $6 \mathrm{p}$ pour 60 toises. Fond en bois, maisons en carton. Assez bien conservé; à réparer.

\footnotetext{
3 The Fortress of Quebec (Québec, 1904), 33-37.

4 Catalogue de plans, cartes et vues ... \$ par P.-L. Morin, copie dactylographiée aux Archives publiques du Canada, 2e partie, 6 .
} 
Enfin un procès-verbal du 12 sept. 1872, signé par le conservateur, M. Le Berryer, signale la démolition d'une dizaine de plans, parmi lesquels figurent ceux de Québec, Montréal et Louisbourg, «complètement hors de service ». Ce document termine donc le dossier et constitue en quelque sorte le certificat de décès de ces plans. Ils avaient subi une longue agonie car, déjà en mauvais état en 1810, ils étaient demeurés encore 62 ans en magasin. Puisque P.-L. Morin avait déjà signalé leur existence en 1854, 18 ans avant leur destruction, on peut regretter que personne au Canada n'y ait porté attention. Il eût été possible de les restaurer et conserver, surtout celui de Louisbourg qui paraissait le moins endommagé. Les Archives publiques du Canada ne fonctionnaient pas encore, mais la Société Historique et Littéraire de Québec existait, et il ne manquait pas d'esprits éclairés parmi ses membres. Au lieu de tomber sous leurs yeux, le rapport de Morin, comme la majorité des rapports officiels, alla dormir dans les tiroirs d'un ministère, puis à la bibliothèque du Parlement, avant d'être versé, tout récemment, aux Archives.

En guise de consolation, on peut se dire que tout n'est pas perdu et que plusieurs plans sur papier subsistent, qui peuvent nous représenter au moins partiellement l'aspect des plans-reliefs perdus.

De Québec, il existe au British Museum un croquis intitulé: «Plan de Québec désigné d'après le plan en relief que M. de Chaussegros de Léry envoya en France en 1720 pour être mis au Louvre avec les autres ». ${ }^{5}$ Ce dessin «fidèlement copié » offre l'avantage de nous révéler l'aspect précis et même les couleurs un peu criardes de l'original. Plusieurs planches gravées de l'époque, très soignées, reproduisent absolument le même tracé et les mêmes légendes. ${ }^{6}$ Elles furent sûrement tirées soit

5 Reproduit dans la $R A C$ pour 1905, I, 4e partie: 20-21.

6 Par exemple le «Plan de la ville de Québec 》 qui figure encore dans le Petit Atlas Maritime de Bellin (1764) I: pl. 9. Voir aussi un plan de Québec, de Chaussegros de Léry, 1722, reproduit par G. Marcel, dans Cartes et Globes ..., pl. 12. 
d'une épure de Chaussegros, soit du plan-relief lui-même. De sorte que nous connaissons à peu près exactement l'état de Québec en 1720.

Le plan-relief de Montréal représentait la ville en 1721, «avec les fortifications projetées et approuvées», c'est-à-dire avant le début de leur construction. Nous ne connaissons pas d'autre plan de cette année. Mais Chaussegros de Léry a laissé toute une série de plans de la ville, de 1716 à 1735 , qui nous montrent ses divers états et son évolution pendant cette période. ${ }^{7}$

Le relief de Louisbourg, non daté mais exécuté vraisemblablement avant 1730 , représentait la ville au début de son histoire. D'après son titre même, cette maquette ne présentait que des «projets de fortifications » qui furent considérablement augmentés et remaniés par la suite. Il existe plusieurs séries de vues, plans et profils, exécutés par les divers ingénieurs en charge, Verville, Verrier, Franquet, et plusieurs plans anglais qui représentent les divers états de la ville et des fortifications dans l'ensemble et les détails, au moment où Louisbourg atteignit l'apogée de sa carrière militaire et maritime, de 1730 à 1760 .

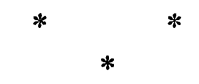

Mais aucun plan sur papier ne peut égaler l'exacte minutie, la fidélité et le pittoresque d'un plan-relief, surtout d'un relief d'époque, exécuté d'après nature, par des ingénieurs-architectes qui étaient souvent des artistes. Un relief montre non seulement les bâtiments militaires et administratifs, mais la topographie des lieux, les magasins des marchands, les maisons des habitants, les places publiques, les arbres, etc. Il donne une représentation des trois dimensions, et restitue une image presque vivante d'une ville. A preuve, l'intérêt que présentent d'autres reliefs de dates plus récentes exposés dans nos musées, par exemple le plan de Québec par Duberger, aux Archives d'Ottawa, et le plan de Louisbourg reconstitué par Miss McLennan.

7 Celui de 1729 a été reproduit par Marcel, ibid., pl. 12, et par C. Bertrand en hors-texte du ler volume de son Histoire de Montréal. 
Le Musée des Plans en relief de Paris demeure une collection unique, très intéressante à visiter. Outre leur valeur intrinsèque, ces plans offrent un intérêt particulier pour le visiteur canadien, par le souvenir de Vauban, dont le système inspira et qui dirigea lui-même quelques-unes des premières fortifications canadiennes. Plusieurs villes françaises y sont représentées, qui eurent des relations avec le Canada, comme Saint-Martin de Ré, Saint-Malo, Belle-Isle-en-Mer, sans parler de la maquette du canal de Panama. Mais le souvenir des trois plans disparus, de Québec, Montréal et Louisbourg, leur causera quand même un nostalgique regret.

RENÉ BAUDRY, Archives du Canada, 1, rue Chanez, Paris. 